\title{
Paradigma eco céntrico en la formación universitaria de la Carrera Ingeniería Ambiental - Facultad de Ciencias Aplicadas, Universidad Nacional de Pilar - Paraguay- 2019.
}

\author{
Ernilda Constancia Vera de Marecos \\ ernildavera@hotmail.com \\ Facultad de Humanidades y Ciencias de la Educación
}

\section{RESUMEN}

La realidad ecológica mundial, pretende un equilibrio aceptable entre la sociedad y el ecosistema natural. Ante estos desafíos actuales, con este trabajo se busca determinar la implementación de los desafíos del paradigma eco céntrico desde la formación universitaria de la Carrera de Ingeniería Ambiental, para encauzar acciones de su implementación erradicando paulatinamente la cultura antropocéntrica. Para el logro de este objetivo se ha desarrollado una investigación acción, desde la perspectiva de dos asignaturas. Se ha realizado una revisión bibliográfica acerca del estado del arte, luego se aplicó fundamentalmente la observación participante, que permitió denotar que la formación universitaria de la Carrera de Ingeniería Ambiental desarrolla el paradigma eco céntrico. La percepción que tienen los estudiantes con respecto a una perspectiva eco céntrica, es aún débil, en cuanto que manifiestan comportamientos antropocéntricos. Mediante propuestas desafiantes, se ha modificado dichos comportamientos, demostrando mayor compromiso con la formación y transferencia de sus conocimientos al ambiente.

Palabras clave: ingeniería ambiental, desarrollo sostenible, cultura antropocéntrica, eco céntrica, ecología. 


\title{
Ecocentric Paradigm in the University Training of the Environmental Engineering Career - Faculty of Applied Sciences, Universidad Nacional de Pilar - Paraguay- 2019.
}

\begin{abstract}
The world ecological reality seeks an acceptable balance between society and the natural ecosystem. Faced with these current challenges, this work seeks to determine the implementation of the challenges of the ecocentric paradigm from the university training of the Environmental Engineering Career, to channel actions for its implementation, gradually eradicating the anthropocentric culture. To achieve this objective, an action research has been developed, from the perspective of two subjects. A bibliographic review about the state of the art has been carried out, then participant observation was fundamentally applied, which allowed to denote that the university training of the Environmental Engineering Career develops the ecocentric paradigm. The perception that students have regarding an ecocentric perspective is still weak, insofar as they manifest anthropocentric behaviors. Mediante propuestas desafiantes, se ha modificado dichos comportamientos, demostrando mayor compromiso con la formación y transferencia de sus conocimientos al ambiente.
\end{abstract}

Keywords: environmental engineering, sustainable development, nthropocentric, ecocentric culture, ecology. 


\section{INTRODUCCIÓN}

Un paradigma es un conjunto de realizaciones científicas universalmente reconocidas que, durante cierto tiempo, proporcionan modelos de problemas y soluciones aceptados por la comunidad científica, según lo expresa Thomas Kuhn en su libro "La Estructura de las Revoluciones científicas" (Kuhn, 1962). Desde ese contexto, hablar de Paradigmas relacionados con la Ecología, constituye toda una filosofía para el abordaje de la Educación Ambiental; porque constituyen maneras de relacionarse con el medio ambiente, sin causar daños al ecosistema, fundamento esencial del Paradigma Eco céntrico. Es menester señalar, que existen dos posturas contrapuestas respecto al Paradigma antropocéntrico; la fuerte y la débil. La primera hace mención a un antropocentrismo egoísta, donde solamente el hombre es el centro, y todo lo que hay en la naturaleza debe estar a su total servicio y la segunda a una acepción más razonable que permite denotar una actitud más emancipadora sobre el ambiente. (Salazar Ortiz \& Láriz Durón, 2017).

El trabajo de investigación planteado contiene diversas teorías relacionadas con el ambiente como la teoría ética ambiental antropocéntrica, la teoría ética ambiental eco céntrica, que, según autores reconocidos, en el área tales como; Esbjörn-Hargens, DiZerega, Kirkman, no deberían ser contrapuestas, sino más bien converger en un paradigma sostenible para el uso adecuado de los recursos naturales provenientes del Planeta Tierra. (cit en Toca Torres, 2011). A partir de estas controversias, surgen otras propuestas teóricas sobre el medio ambiente, éstas constituyen las teorías globalizantes, o biologuitas, que intentan incorporar un altruismo biosférico, tendiente a la protección de otras especies o hacia el uso de los recursos naturales de manera armónica, más allá de los beneficios egoístas de los humanos. (Oliveros, 2915).

El antropocentrismo es una postura respecto a la acción sobre la naturaleza, que ubica al hombre en el centro del medio ambiente, como dueño y poseedor del mismo; bajo la cual han nacido normas, corrientes ideológicas, posturas políticas, que asiente al ser humano la potestad de manipular el medio ambiente hasta el punto de destrozarlo, sin reparar en que el deterioro causado a la Tierra desembocará tarde o temprano en un daño a su buen vivir en este Planeta. (Hernández Islas, 2020).

Al respecto, Gabriela Hernández, considera lo siguiente: 
El pronóstico científico nos señala que llegaremos a un punto en el que ya no habrá retorno, nos señala que estamos en el camino hacia ningún lugar. Desbancar el antropocentrismo como eje central de la ideología humana conlleva replantearnos cuestiones éticas de carácter histórico a la luz de nuevos elementos de juicio. El tema de la interrelación hombre-medio ambiente es uno de los asuntos que debe analizarse tomando en cuenta todos los elementos (no únicamente al ser humano y sus intereses). (Hernández Islas, 2020, párr. 5.).

Existe un sinnúmero de organizaciones que se han preocupado desde mediados del siglo $\mathrm{XX}$, acerca del uso racional de los recursos naturales y de la práctica genuina de la Educación ambiental, en el mundo. Estas acciones internacionales se originaron en Francia en el año 1948, con la Unión Internacional para la Conservación de la Naturaleza (UICN); seguidos por la Organización de las Naciones Unidas para la Educación, la Ciencia y la Cultura (UNESCO), que en el año 1949, realiza la primera investigación internacional de cómo estudiar la naturaleza con fines educativos.+

La Ecología se halla en una situación crítica a nivel mundial, pareciera que a nadie le interesa tal situación, sin embargo, estudios realizados respecto al deterioro del ambiente revelan que:

Actualmente, la humanidad se enfrenta a una serie de problemas globales que dañan la biosfera y la vida humana de modo alarmante y que podrían convertirse en irreversibles. La publicación del famoso informe "Los Límites del Crecimiento", encargado en 1972 al Instituto Tecnológico de Massachusetts 4 (MIT, siglas en inglés de Massachusetts Institute of Technology) por el Club de Roma, concluía afirmando que si el actual incremento de la población mundial, la industrialización, la contaminación, la producción de alimentos y la explotación de los recursos naturales se mantenía sin variación, alcanzaría los límites absolutos de crecimiento en la Tierra durante los próximos cien años. Sin embargo, conforme el Informe Planeta Vivo 2014 de la Fundación Vida Silvestre (Word Wild Fund, WWF, por sus siglas en inglés), hay un déficit planetario del $40 \%$, que se estima alcanzará el 100\% para mediados de 2030.

Los actuales patrones de consumo mundiales son insostenibles. Los niveles de consumo globales son impulsados por el rápido crecimiento de la población mundial que se triplicó en los últimos 100 años y se espera que aumente un $30 \%$ más en los próximos 35, llevando a la población a 9 mil millones para el año 2050 (Martínez \& Porcelli, 2018, 1er párr). 
Estos resultados alarmantes deberían crear espacios de reflexión acerca de los fenómenos globales que generan esta situación, y la mejor manera de hacerlo es al interior de las universidades, donde se generan los conocimientos, y se crean conciencia positiva hacia el cuidado del ambiente y el manejo sostenible de los recursos naturales.

Asimismo, existe un estudio realizado en tres países de Iberoamérica, respecto a la concepción que los estudiantes universitarios tienen acerca de las Representaciones Sociales(RS) del Medio Ambiente. "Las representaciones sociales permiten a los sujetos dar sentido a la compleja realidad en que transcurre su vida cotidiana. Estas representaciones se comparten con otras personas al interactuar, lo que les confiere un carácter intersubjetivo" (González Gaudiano \& Valdez, 2012, pág 4).

El primer paso para iniciar cualquier trabajo de Educación Ambiental, de acuerdo con la perspectiva de las representaciones sociales, consiste en identificar las RS que tienen las personas involucradas en los procesos educativos. Es así que Ferrerira (2002), pone en relieve algunos estudios realizados sobre representaciones sociales del Medio Ambiente, conforme a la tipología estudiada por Reigota (1990), y encontró que, entre las naturalistas, que evidencian los elementos naturales y los seres vivos; las antropocéntricas, que presentan la utilidad de los recursos naturales para la sobrevivencia de los humanos; y por último las globalizantes, que involucran aspectos naturales y sociales. En este sentido, la mayoría de los estudios realizados en el ámbito de Brasil, ya sea con docentes o estudiantes, demuestran una predominancia de posturas naturalistas. Asimismo, Andrade en otro estudio realizado con estudiantes del profesorado, ha encontrado que las RS de los estudiantes analizados muestran una incoherencia entre el pensar y el hacer pedagógico; se detecta, además, que la transversalidad es un tema aún oscuro, para estos futuros docentes. (González Gaudiano \& Valdez, 2012).

Andrade (2004), analizó las RS de 204 alumnos universitarios en Río de Janeiro, en el que identificó tres categorías para el medio ambiente: antropocéntrica utilitarista, antropocéntrica pactuada y eco céntrica. El resultado de este estudio derivó en el predomino de la categoría antropocéntrica "pactuada", en la cual el hombre sí es visto como elemento central del ambiente, pero sus objetivos tienden a ser, en lo posible, armonizados con la naturaleza. Estos resultados sobre las representaciones sociales son consistentes hacia una visión naturalista, según otros investigadores como Molfi Goya (2000), Azevedo (1999) y Okamura (1996), quienes también han encontrado la 
predominancia de las RS, sobre la tipología Naturalista de Reigota (como se citó en González Gaudiano \& Valdez, 2012).

Estas percepciones acerca de la visión Medio ambiente, denotan que es necesario una clarificación de ideas respecto al MA, en las instituciones de Educación Superior y las universidades

También en este artículo de revisión se ha presentado las investigaciones realizadas en México en distintos niveles educativos, en lo que respecta a la formación de profesores de educación primaria, al respecto, Terrón (2009) encontró que las RS, se caracterizaban como Reducidas o simples, que entienden al ambiente como sinónimo de naturaleza, medio físico; la problemática ambiental se restringe al deterioro ecológico, y los individuos recurren a actividades puntuales para su protección y cuidado; otra categoría hallada fueron las Gobalizadoras, que demuestran el valor contenido en los ciudadanos para solucionar los problemas ambientales. Las Antropocéntricas técnicas, que confiere compatibilidad entre el progreso y la preservación del medio ambiente y que mediante el uso racional de la tecnología y la ciencia, se puede mejorar el ambiente, incluso revirtiendo algunos daños ambientales; por último están las Sociales integrales, en las que se interrelacionan los aspectos físicos y sociales de la problemática ambiental, albergando un sentido humanista de la EA, que busca el bienestar social y el equilibrio con la naturaleza y una mejor convivencia entre las sociedades (González Gaudiano \& Valdez, 2012)

Estos resultados, en general son poco alentadoras, respecto a países donde el tratamiento de estos temas ya se supone han sido consolidados; sin embargo, podemos notar que la mayoría de los resultados evidencian una visión atomizada y antropocéntrica sobre el Medio Ambiente, con algunas excepciones cuando se trata de la formación para profesorados.

Lo referente a estudios realizados en España, respecto a RS, se ha encontrado que los estudiantes universitarios confieren el problema del ambiente al cambio climático generado a raíz de dos modelos principales, de la acción antrópica; “ el hombre emite contaminantes a la atmósfera y la naturaleza responde con el cambio climático; el segundo, la contaminación produce un "agujero" en la atmósfera, por el cual entran más rayos solares produciendo el calentamiento global" (Meira, como se citó en González Gaudiano \& Valdez, 2012, p. 12). 
El antropocentrismo es una postura filosófica que, según Hernández, se ha ido gestando luego del teocentrismo, con el propósito de mejorar la condición humana y concibe al ser humano y sus intereses como el centro de todo, por lo que se produce una supeditación de los demás seres vivos, medio ambiente, etcétera; a las necesidades y bienestar del ser humano. Esta Teoría filosófica se engendra con Francisco Bacón, Francois Ost (1996), que expresa lo siguiente: "el Estado moderno debe concebirse como una república científica $[\ldots]$ cuyo objetivo es llegar a un dominio completo de la naturaleza con vistas a mejorar la suerte del género humano", (pág 34) (cit en Hernández, 2020; 5to.párr.). Con estas expresiones, se gesta la visión de que el hombre es el dueño y señor de la naturaleza y que debe hacer uso de ella a sus antojos.

Las teorías relacionadas con el antropocentrismo, se desprende de una visión egoísta del hombre, pues deriva de la raíz etimológica:

Antro $=$ hombre $;$ centrismo $=$ centro

En esta vertiente se considera al hombre como centro de todo lo creado, una corriente donde se afirma que el hombre es el centro del cosmos y, por lo tanto, la interacción entre el hombre y los recursos naturales siempre debe favorecer al hombre o rondar en torno a él; por el otro lado, tenemos una corriente más conservacionista que nos dice que el ambiente es el centro del cosmos y, por tanto, el hombre es solo un ingrediente dentro de la gran interacción de la vida. (Mantilla Oliveros, 2015, p.427).

$\mathrm{El}$ antropocentrismo se centra en la creencia de que los humanos son superiores al resto de la naturaleza, por lo que, como resultado, se considera al ser humano como legítimo dueño de aquella y, por ende, puede utilizarla para sus propósitos, de modo que la naturaleza tiene un valor por su contribución a la calidad de la vida humana, satisfaciendo sus necesidades físicas y materiales; en sentido contrario, la visión ecocéntrica considera que la naturaleza contiene un valor inherente, independientemente de si le es de utilidad o no al ser humano; en este sentido los ecocéntricos valoran a la naturaleza por sí misma. (Lorenzzetti, 2008) y (Figueroa, 2014).

Por las reflexiones expuestas, en este artículo, se pretende describir en qué medida la formación universitaria de la Carrera de Ingeniería Ambiental desarrolla el paradigma ecocéntrico; además de revisar sus representaciones sociales sobre el ambiente, que constituyen un parámetro fundamental, para el aprendizaje de los estudiantes, de modelos 
de representaciones ecológicas. Asimismo, se busca demostrar que existen estrategias pertinentes que tienden a mejorar las percepciones de los mismos, respecto al ambiente.

\section{ESTRATEGIAS METODOLÓGICAS O MATERIALES Y MÉTODOS}

Para este estudio, se ha abordado una Investigación Acción; con componente cuali y cuantitativo, ya que durante el proceso de enseñanza aprendizaje, se han incorporado estrategias que permitieron la movilización de las estructuras cognitivas y afectivas de los estudiantes, con relación a la implementación del Paradigma Eco céntrico. Se ha tomado este diseño de investigación teniendo en cuenta que los cambios son más significativos, y efectivos cuando los miembros de estos grupos se implicaban en los procesos de investigación y toman parte colectivamente en las decisiones sobre los cambios oportunos (Contreras, 1994). Así también Ander-Egg (2003), plantea que este tipo de trabajo, demanda un proceso reflexivo, sistemático, controlado, y de intervención, cuyo fin es estudiar un aspecto de la realidad para modificarla. Y según el trabajo realizado, los resultados arrojados de esta reflexión sistemática, serán utilizados para mejorar la posición de los futuros ingenieros ambientalistas con respecto a estas percepciones sobre el ambiente, que conlleva grandes ideologías, filosofías y teleologías respecto a la relación del hombre con la naturaleza. El estudio incluye aspectos del tipo descriptivo con componente cualitativo y cuantitativo; pues utilizando diversos procedimientos para el levantamiento de datos como el análisis documental, el cuestionario y las entrevistas a informantes claves, se han ido caracterizando la actitud de los estudiantes, respecto al objeto de estudio planteado. Para el logro de los objetivos planteados, se llevó a cabo una metodología de InvestigaciónAcción, que ha permitido avizorar el comportamiento de los estudiantes dentro del aula, conforme a estrategias planteadas. Se aplicó un cuestionario estructurado, validado por (Fernández Manzanal, Pérez de Heredia, Rodríguez Barreiro, \& Marcén Albero, 2003) y ampliado para complementar ellas características del estudio, que ha permitido denotar su actitud ante la teoría antropocéntrica o eco céntrica acerca del manejo del ambiente, ese resultado permitió implementar otras actividades desafiantes para modificar las actitudes de los mismos ante el enfoque antropocéntrico, para lograr una visión más eco céntrica. Este cuestionario se ha aplicado a los estudiantes de las cátedras de Ecología General y Urbana, Ecología de Humedales y Pastizales, alumnos del segundo y tercer curso de la Carrera de Ing. Ambiental. El criterio de inclusión lo constituyeron todos los alumnos que se han inscriptos en dichas cátedras durante el año 2019. Y los de exclusión se consideran 
algunos alumnos que se nieguen a participar activamente de algunos eventos relacionados con la investigación. Se ha utilizado la Observación Participante, considerando que se desarrolló en el aula, en interacción con los estudiantes. Esta técnica aborda aspectos conceptuales, actitudinales y conativos, lo cual permite incorporar acciones o metodologías que propendan el mejoramiento de los procesos desarrollados en el espacio de observación (Vieytes, 2004).

En primer lugar, se realizó el diagnóstico, mediante la aplicación del cuestionario, que permitió identificar la actitud de los estudiantes respecto al paradigma eco céntrico o antropocéntrico que practican en su cotidianeidad.

Se realizó, además, entrevista a informantes claves, los que constituyeron los docentes de la Carrera, y los responsables de Investigación y Extensión. El análisis documental, sirvió para identificar las políticas desarrolladas para esta visión eco céntrica o antropocéntrica, abordada desde la UNP, y la carrera de Ingeniería Ambiental. El cuaderno de campo permitió registrar las actividades desarrolladas en el marco de la ejecución el proyecto de investigación, con los estudiantes involucrados en el estudio. Mediante esta técnica se pudo registrar los aspectos conceptuales y actitudinales de los estudiantes, respecto al medio ambiente, esto sirvió de sustento contextualizado para implementar las estrategias en el campo de la acción, con enfoque más holístico, y emancipador en el ámbito de la sustentabilidad de los recursos.

Para el análisis de los resultados se ha utilizado el propuesto por Stenhouse, que el desarrollo profesional de los profesores depende de la capacidad para adoptar una postura investigadora en relación con su ejercicio docente, en el ámbito de la Investigación- acción. En ese contexto, se llevó un control estricto de las reflexiones, que surgieron de las discusiones desarrolladas en los grupos de trabajo. A partir de esas percepciones y concepciones, se implementarán los ajustes relacionados con el paradigma deseado, para implementar dicha cultura al interior de los grupos de estudiantes y docentes; luego al cierre de esta investigación, se analizan los cambios, los logros para que finalmente los conocimientos y aprendizajes, sean planteadas a la institución para derivar en políticas educativas para la Carrera y la Comunidad. 


\section{RESULTADOS Y DISCUSIÓN}

Los resultados de esta investigación se sistematizan en cuatro apartados fundamentales que hacen referencia al resultado de una encuesta inicial aplicada a los estudiantes, otro apartado comprende el resultado de portafolios de evidencias desarrollado con los estudiantes en las cátedras respectivas para avizorar sus conocimientos y su visión respecto a las concepciones acerca del ambiente; otro apartado contiene el análisis documental referido a los documentos institucionales y por último se analizaron las percepciones de los docentes respecto a la misma visión.

\section{El Diagnóstico}

Se aplicó un cuestionario estructurado a 48 estudiantes de la Carrera de Ing. Ambiental, en relación con sus percepciones, respecto a seis categorías relacionadas con la Educación Ambiental.

a) El primer criterio alude a la cantidad de basuras y de residuos como problema ambiental, se ha encontrado que el $85 \%$ se molestan ante las basuras dispersas; el 50\% consideran que la acumulación de basuras no constituye un problema, el 35\% cree que el problema mayor es el lugar para ubicarlos; el 45\% no está de acuerdo, y el resto se mantiene con una postura indiferente; el $71 \%$ consideran que se acumulan basuras por causa de la consumición innecesaria. Estos resultados develan que las RS de los alumnos convergen en las globalizantes, pues perciben que lo social tiene mucha influencia en la acumulación de las basuras, como problema ambiental.

b) El consumo y su relación con la producción de residuos, los resultados de este criterio ha permitido demostrar que un $67 \%$ prefieren escoger productos con envoltorios más amigables, un $40 \%$ ha considerado que no es malo consumir mucho, ya que gracias a eso se brindan puestos de trabajo; un 52\%, está de acuerdo con la cultura de usar y tirar; más del $50 \%$ considera que su aportación en ahorro de recursos, a costa del bajo consumo, es insignificante; asimismo, un $50 \%$ ha confirmado que no se halla dispuesto a reducir el consumo. Estos resultados derivan en una postura más o menos naturalista y antropocéntrica, pues un poco más de la mitad de los consultados consideraron bueno reducir la producción de residuos al momento de consumir; no obstante, un buen porcentaje minimiza la importancia de la relación que hay entre el consumo y la acumulación de los residuos. 
c) La Gestión y el coste de residuos, se ha encontrado que el $73 \%$ de los estudiantes consideran que aquel que contamina es quien debe pagar; y que los usuarios no consideran el abultamiento de los residuos al utilizar los productos desechables; el 85\% está de acuerdo con la utilización del papel reutilizado. El principio de: “quien contamina paga”, surgió en la Organización para la Cooperación y el Desarrollo en Europa (1972), su objetivo fue de localizar los costes de la prevención de la contaminación y las medidas de control de las mismas, para asegurar un uso racional de los recursos naturales. (García Amez, J. cit. en Oliveira, 2017). Aquí vemos que un elevado porcentaje de estudiantes están de acuerdo con la aplicación de este principio, el cual permite inferir que éstos se inclinan hacia posturas antropocéntricas técnicas, donde el uso de los recursos naturales debe ir acompañado de la utilización razonable de la tecnología, como medio de minimizar el deterioro ambiental.

d)El reciclaje y la reutilización, la mayoría de los estudiantes consideraron muy interesante aplicar el reciclaje y la reutilización de los productos que se consume, en este caso nuevamente éstos se inclinan hacia posturas globalizantes; sin embargo, no están interesados en adquirir mayor información sobre el reciclado de basuras, en ese sentido, sus respuestas son un tanto inconsistentes.

e) Evaluando la recogida selectiva, se ha encontrado que más del $50 \%$ no consideran oportuno llevar las bolsitas de polietileno; el $80 \%$ considera que no se ha insistido bastante aun en la importancia de separar las basuras, el 83\% cree oportuno usar basureros diferenciados; sin embargo, un 35\% se ha mantenido indiferente, respecto a la separación de basuras en sus casas en contenedores diferenciados. Ha existido una actitud de indiferencia ante la recogida selectiva, en un porcentaje elevado, lo cual permite denotar una postura antropocéntrica, considerando que minimiza la ingente cantidad de basuras que se genera a causa de la disposición de los mismos en un solo contenedor.

f) Con relación al ahorro de energía, el $83 \%$ de los alumnos consideraron que plantando un árbol colabora con la reforestación, un porcentaje similar, $81 \%$ está de acuerdo que administrando el consumo de agua colabora con el ahorro de energía; un $60 \%$ se ha posicionado ante la postura de que normalmente le preocupa que en la casa se hallen conectados, aparatos y luces prendidas; con respecto a si creen importante la evaluación del impacto ambiental, el 80\%, han considerado que sí es importante. Estos resultados permiten denotar que poseen una actitud bastante favorable respecto al ahorro de energía, lo cual 
confiere una postura globalizante, considerando que el ciudadano es el responsable de solucionar los problemas ambientales.

A estos resultados se ha sumado las observaciones de clases en los grupos, las entrevistas a informantes claves y el análisis documental de los documentos estratégicos como el Proyecto Educativo Institucional y el Proyecto de Carrera.

El resultado de las observaciones permitió denotar que éstos consideran que los actores ideológicos y políticos poco tienen que ven en los problemas ambientales; lo referido permite aseverar que existen más implicancias de una cultura antropocéntrica débil, aunque sutilmente se observa en algunos grupos la visión naturalista y globalizante, con respecto a las teorías que sustentan el medio ambiente, conforme a lo planteado por Ost (1996), quien concibe a la naturaleza como un sistema, en el que todo cuanto perviva en ella se deben relacionar armónicamente; considerando los innumerables intercambios físicos, químicos, energéticos y biológicos, que se establecen en el seno de los ecosistemas y desarrollan el fenómeno dinámico de la naturaleza.

Asimismo, los documentos analizados, permitieron demostrar que existen ejes estratégicos que permitirían coadyuvar con la incorporación de una visión más globalizante; no obstante, conforme a las entrevistas realizadas con informantes claves se ha podido denotar que se tienen en los documentos, pero la manera de encararlos es un tanto limitante. Asimismo, también los encargados de los programas de investigación y extensión han manifestado que existen escasos proyectos relacionados con la Educación ambiental y el desarrollo sustentable, lo que sugiere el monitoreo $\mathrm{y}$ fortalecimiento de dicha implementación.

Estos hallazgos permiten deducir que los estudiantes poseen representaciones sociales(RS) ambientales bastante aceptables, comparándolas con estudios realizados por investigadores latinoamericanos, acerca de la visión del medio ambiente, en la que hallaron tres categorías; la antropocéntrica utilitarista, la antropocéntrica pactuada y la eco céntrica; en general, los hallazgos en este trabajo con los estudiantes, se inclina hacia la segunda y la tercera categoría de las RS.

Estos resultados permiten ahondar en la reflexión acerca de la necesidad de implementar estrategias que conlleven a comprometer a estos estudiantes con su responsabilidad social como universitarios acerca de la solución de los problemas ambientales aparejados con la postura social antropocéntrica imperante en el medio social. Para responder a dicha 
necesidad, se ha diseñado estrategias que se enmarcan dentro de planteamientos más desafiantes y comprometidos por parte de los alumnos con la incorporación de una cultura más ecocéntrica-biocéntrica, durante su proceso de formación académica.

\section{Implementación de las estrategias}

En proceso de intervención con estrategias innovadoras, conforme a los resultados hallados hasta el momento se han realizado las siguientes intervenciones desde la práctica, con el propósito de mejorar sus percepciones sobre el entorno ecológico natural. Algunas de las actividades que propiciaron suficiente compromiso de parte de los alumnos que han participaron fueron:

a) Las salidas de campo.

Estas salidas de campo permitieron identificar los tipos de vegetación de los humedales, esto lo hicimos en los ecosistemas urbanos y en ecosistemas rurales para ver si esta vegetación igual se adaptaba a aguas contaminadas de la ciudad, lo que arrojó un resultado donde se pudo observar que las vegetaciones en los ecosistemas urbanos no podían desarrollarse como en un ecosistema que se encontraba lejos de la ciudad, eso ha salido como reflexión de cómo el ecosistema urbano es diferente al rural por la acción antrópica que se evidencian en esos contextos.

b) Audición radial

Para esta actividad, los estudiantes tuvieron que dotarse de los conocimientos pertinentes acerca de los temas ambientales, relacionados con la visión antropocéntrica y ecocéntrica, para poder informar con terminología técnica perspicaz, los hallazgos que han tenido en sus salidas de campo. Uno de los trabajos presentados fue el trabajo de investigación realizado acerca del vertedero local, al cual la Municipalidad de Pilar lo nomina "Relleno Sanitario"; terminología que no le corresponde, por no adecuarse a los requerimientos mínimos exigidos para dicha denominación. Es interesante recalcar que los estudiantes con este tipo de actividades, se sintieron mucho más comprometidos con la investigación bibliográfica, para contemplar todos los requerimientos teóricos que necesitan para enfrentarse a este tipo de retos. Con este trabajo de investigación se pudo detectar la contaminación del agua, del aire y del aire. El agua y el suelo se contaminan por el lixiviado de las basuras arrojadas sin ningún tratamiento en dicho vertedero y el aire a través de las quemas de los residuos sólidos. De este trabajo de investigación se tuvo el siguiente resultado: que la ciudadanía en su mayoría desconoce la diferencia entre vertedero y relleno sanitario. Y que se debe 
hacer un trabajo de concienciación para que cada ciudadano realice la separación de los residuos en sus respectivos hogares. Que los camiones recolectores salgan en días diferentes para la recolección de los residuos. Con este trabajo se notó el compromiso de los estudiantes y las ganas de aportar para mejorar la condición de vida de todos los habitantes de la ciudad de Pilar, fue un logro positivo desde esta cátedra, que ha impactado en la comunidad.

c)Interacción en espacios de presentación de trabajos.

También hemos invitado a ex alumnos del Instituto de Ciencias Ambientales a presentar sus trabajos de investigación sobre diferentes temas ambientales. Esta audición radial fue conducida por los estudiantes del 2 do curso de esta carrera, lo que permitió un involucramiento positivo con la situación medioambiental de la Ecorregión.

d)Proyección hacia la comunidad con trabajos de extensión

Este trabajo fue de índole interinstitucional, entre el Instituto de Ciencias ambientales y los colegios secundarios para recolectar pilas y enseñar a la comunidad el grado de toxicidad de las pilas para el medio ambiente, se realizaban la recopilación de las pilas, y en simultáneo los estudiantes, desarrollaban las charlas pertinentes de cómo afecta la contaminación por este contaminante. Se aclara que, para la realización de este tipo de charlas, nuevamente los estudiantes debían estar bien preparados para el desarrollo de estas charlas. Los mismos estudiantes se encargaban de explicar a la ciudadanía acerca de este tema.

e) Trabajos de investigación

Esta modalidad de trabajo consistió, que previamente a un estudio en referencias teóricas acerca de la vegetación presentada por los Humedales en general; fueron formando grupos para investigar in-situ, acerca de la vegetación con que se cuenta en los Humedales del Ñeembucú. Otras investigaciones realizadas fueron la de caracterizar las distintas Ecorregiones del Paraguay y en especial la Ecorregión de Ñeembucú acerca de su flora, fauna y grado de contaminación de las aguas del ecosistema urbano, entre otros. Se realizó una excursión para identificar in-situ, la Ecorregión de Ñeembucú. Otras investigaciones desarrolladas fueron dimensionar y reflexionar acerca del impacto negativo ocasionado por las obras de infraestructuras sobre el medio ambiente y sus medidas de mitigación. Estos trabajos permiten sensibilizar a los estudiantes hacia la realidad ecológica circundante, lo 
cual favorece su compromiso como futuro profesional para intervenir en la dotación de soluciones a los problemas ambientales, productos de la acción antrópica.

De esta manera se percibe que, a través de estos desafíos, se produce la modificación las actitudes y percepciones de los estudiantes, respecto a esta visión ética comprometida con el ambiente natural, articulándose en sus percepciones, de esta manera la visión ecológica globalizante.

\section{Otras estrategias implementadas para mejorar la percepción de los estudiantes respecto a la visión eco céntrica sobre el medio ambiente}

En el marco de la internalización del modelo eco céntrico, los alumnos participaron de varias actividades de protección al medio ambiente tales como: limpieza de las costas del arroyo Neembucú, elaboración de colectores de basuras para el Instituto, se trabajó con un proyecto interinstitucional de recolección de pilas usadas para evitar que se tiren al suelo por el alto grado de contaminación que produce, se colectaron varias toneladas de éstas, que fueron llevadas a la Secretaría del Ambiente (SEAM) a la capital del país por la Gobernación del Departamento, el proyecto incluyó a Instituciones del nivel medio y al Instituto de Ciencias Ambientales.

Para propender la sensibilización hacia la visión eco céntrica, y a través de la Materia: Ecología General y Urbana, se ha hecho un proyecto de relleno sanitario para la ciudad de Pilar; se ha presentado al municipio para trabajar en forma conjunta, con los alumnos, éstos se han preparado para desarrollar capacitaciones y concienciación a la población en forma gratuita para que el municipio ponga la parte económica, y así mejorar el actual vertedero y convertirlo en un relleno sanitario para salvaguardar la salud de la población. Pero este proyecto, que de por sí, ya ha constituido un aprendizaje para los estudiantes; sin embargo, no ha tenido una aceptación favorable por parte de los intendentes de turno.

Se realizó actividades de reciclaje para que los alumnos valoren que con este tipo de actividad se protege al medio ambiente.

Los alumnos participaron del proyecto "Basura cero", propulsada por otras organizaciones. También se realizaron charlas en los colegios por parte de los estudiantes de Ing. Ambiental, acerca de Protección del medio ambiente. 


\section{Evaluación de las estrategias implementadas}

Luego de estas actividades, se les ha consultado a los estudiantes, su percepción respecto al ambiente. A continuación, se presentan algunas observaciones dadas por los involucrados

-Este tipo de estrategias nos resulta más significativas, porque forma parte de lo que genuinamente realizaremos cuando seamos profesionales.

-Este tipo de contacto nos hace notar la importancia de una visión más eco céntrica, además hemos notado la indiferencia, en algunos casos de las autoridades, para propiciar cambios con relación a la visión antropocéntrica, que aún persiste fuertemente en nuestra sociedad. -Así aprendemos más significativamente, y adquirimos capacidades de relacionamiento con las personas involucradas con las actividades ambientales.

-Es muy importante desarrollar este tipo de actividades porque nos hace vivenciar el entorno, y la necesidad de trabajar con mayor empeño en proyectos ecológicos.

-Son muy valiosas las estrategias propuestas por la asignatura, y aprendemos contenidos y procedimientos para actuar y relacionaros con los demás.

-Aprendemos a realizar los proyectos ecológicos, y a participar de otros desarrollados en la comunidad.

-Es inagotable lo que hoy día se puede hacer por el ambiente.

Se ha visualizado, además que los estudiantes asumieron un mayor compromiso a la hora de abordar los temas propuestos desde la asignatura, proponiendo sugerencias de salidas de campo e intervenciones a nivel comunitario. Surgieron como propuestas de trabajos ambientales, algunos proyectos de selección y recogida de basuras en algunos barrios de la ciudad de Pilar, a iniciativa de los estudiantes. Estas respuestas han estimulado más para seguir trabajando en el ámbito de crear conciencia positiva hacia el ambiente en los futuros graduados de Ingeniería Ambiental, pues ellos serán los que en el futuro podrán modificar la actitud antropocéntrica hacia el ambiente, y desarrollar más actitudes ecocéntricas o globalizantes.

\section{CONCLUSIÓN O CONSIDERACIONES FINALES}

Luego de una revisión bibliográfica exhaustiva de los teóricos que fundamentan la visión eco céntrica y antropocéntrica de la Educación Ambiental y considerando la triangulación de distintas fuentes se destaca la persistencia de una actitud más o menos positiva, respecto a la contaminación; no obstante, perviven culturas antropocéntricas, 
globalizantes y antropocéntricas-técnicas, respecto a algunos problemas ambientales. No se ha podido verificar una actitud decisiva e impetuosa acerca de su intervención en la mejora de los problemas ambientales mediante proyectos de intervención socialmedioambiental. Se considera que esta actitud responde al abordaje teórico, poco práctico de los problemas ambientales; esta aseveración se atribuye a la escasa existencia de proyectos ambientales trabajados con el medio social, propiciado por docentes y estudiantes, lo que conlleva a inferir que falta mejorar las estrategias para asumir compromisos que conlleven a la intervención en la mejora de los problemas ambientales del entorno inmediato.

Existen posturas que favorecen el mejor tratamiento para minimizar el desgaste de la materia prima, que consiste en el reciclado; estos estudiantes están de acuerdo con esta postura; no obstante, una mayoría no se halla muy interesado en adquirir más conocimientos sobre estas estrategias de reciclado. En ocasiones, también existen confusiones respecto a lo que significa reciclado y reutilizado, que no son conceptos sinónimos. Una postura bastante interesante respecto a la visión eco céntrica lo constituye la separación de las basuras en contenedores diferenciados, mínimamente en dos tipos de residuos, los estudiantes demuestran no tener dicha práctica en sus vivencias cotidianas, es que no existen políticas institucionales, ni municipales para dicha práctica en la ciudad. Este es una deuda aún que se tiene respecto al cuidado del ambiente, y es una manera de recuperar mucha materia prima útil. No obstante, con las estrategias implementadas, a posteriori, se ha podido modificar la actitud hacia esos procesos, porque han vivenciado en la comunidad la necesidad de mejorar dichas conductas, de manera contextualizada y globalizante.

En lo que respecta al ahorro de energía, los estudiantes manifiestan una actitud bastante positiva y altruista respecto a su utilización, y demostraron poseer una concienciación oportuna en la economía de su uso.

Existen políticas institucionales que coadyuvan con la implementación efectiva de los problemas ambientales, enunciados en la misión y visión respectivas; sin embargo, es necesario articular con los procesos académicos, de tal modo a operacionalizarlos por diversos procesos desarrollados en las prácticas de aula, y su transferencia efectiva al entorno inmediato. 
No se evidencia en sus comportamientos un deseo de mejorar la situación ecológica del entorno, con la escasa presentación de proyectos relacionados con la mejora del entorno ecológico; esas y otras actitudes permiten deducir que aún persisten comportamientos relacionados con una cultura antropocéntrica en los estudiantes de la Carrera Ingeniería Ambiental de la FCA de la Universidad Nacional de Pilar.

En función a los resultados obtenidos de este trabajo de investigación, y los planteamientos trabajados con los estudiantes, durante el desarrollo de la misma, se plantean las siguientes recomendaciones a los distintos estamentos:

-A los responsables de la Gestión, se sugiere que los ejes propuestos para el tratamiento de proyectos ambientales sean monitoreados desde un proceso de evaluación sistemático para realizar los ajustes de la implementación a través de las funciones sustantivas: docencia, investigación y extensión.

-A los responsables de la Carrera y de las funciones de Investigación y Extensión. Monitorear el cumplimiento de los propósitos sobre la Educación Ambiental descriptos en los programas de estudios. Verificar que la intervención didáctica sea teórica y práctica, de tal modo que los estudiantes adquieran herramientas propicias para la formulación de proyectos ambientalistas, que propendan una visión crítica sobre los problemas ecológicos, y la acción antrópica. Propender actividades de docencia, investigación y extensión que propulsen el cuidado del entorno ecológico, donde sean involucrados docentes y estudiantes. Transferir a la comunidad los resultados de dichas investigaciones a través de diversos medios, para que, mediante esas difusiones, tanto los estudiantes involucrados, como la comunidad, tome conciencia de la visión ecocéntricaglobalizante necesaria para asegurar la sostenibilidad de los recursos naturales.

-A los colegas docentes, se sugiere la implementación con mayor sistematicidad los Proyectos que tiendan al Desarrollo Sustentable, y a una visión eco céntrica, que involucre los estudiantes, además de eso la realización de propuestas didácticas, innovadoras, que constituyan más al hacer y al ser para que los estudiantes se sientan involucrados con las actividades de planificación, ejecución y evaluación de proyectos ambientales. Proponer la realización de investigaciones de campo, respecto a las capacidades planteadas en los programas de estudios, de tal modo que los estudiantes adquieran competencias ecológicas con una visión ecocéntrica- globalizante. 
-A los estudiantes. Realizar investigaciones relacionadas con el Paradigma ecocéntricoantropocéntrico- globalizante. Aceptar los desafíos propuestos por los docentes con espíritu crítico y emprendedor.

\section{LISTA DE REFERENCIAS}

Ander-Egg, E. (2003). Repensando la Investigacion Acción Participativa. Comentarios, críticas y sugerencias. Buenos Aires: Lumen.

Ávila, P. Z. (2018). La sustentabilidad o sostenibilidad: un concepto poderoso para la humanidad. Tabula Rasa.(28), 409-423.

CEPAL. (2016). Agenda 2030 y los Objetivos de Desarrollo Sostenible. Una oportunidad para América Latina y el Caribe. Santiago: publicaciones@ cepal.org.

Contreras, J. (1994.). La investigación en la acción ¿ qué es ? ,. Cuadernos de Pedagogía $N^{\circ} 224$.

Fernández Manzanal, R., Pérez de Heredia, A. H., Rodríguez Barreiro, L. M., \& Marcén Albero, C. (2003). Educación Ambiental. Ecosistemas. Revista Científica y Técnica de Ecología y Medio Ambiente, 1-18.

Figueroa, A. O. (2014). Medioambiente como Bien Jurídico PROTEGIDO, ¿VISIÓN ANTROPOCÉNTRICA O ECOCÉNTRICA? REVISTA DE DERECHO PENAL Y CRIMINOLOGÍA, 253-294.

Flores, R. C. (2018). Una Experiencia En Educación Ambiental Con Estudiantes Universitarios. Journal of Sustainability Education, 18, 1-15.

Facultad de Ciencias Aplicadas. Universidad Nacional de pllar. (2018). Proyecto educativo. Actualización del Proyecto Educativo de la Carrera Ingeniería Agropecuaria de la UNP. Pilar, Paraguay: Universidad Nacional de Pilar.

González Gaudiano, E., \& Valdez, R. E. (2012). Enfoques y sujetos en los estudios sobre representaciones sociales de medio ambiente en tres países de Iberoamérica. Revista de Investigación Educativa 14, 1-17. Obtenido de http://www.uv.mx/cpue/num14/inves/gonzalez_

Hernández Islas, G. (02 de enero de 2020). Recuperado el 08 de 06 de 2020, de https://forojuridico.mx/la-vision-antropocentrica-proteccion-y-derechos-del$\underline{\text { medio-ambiente/\#_ftn1 }}$

Kuhn, T. S. (1962). La Estructura de las Revoluciones Científicas (2004 ed.). (A. Contín, Trad.) México. 
Lorenzzetti, R. L. (2008). Teoría del Derecho Ambiental. México: Porrúa, SA de CV.

Mantilla Oliveros, N. A. (2015). Reformulación del principio de antropocentrismo ambiental. Justitia, 421 - 439.

Martínez, A. N., \& Porcelli, A. M. (2018). Del antropocentrismo al ecocentrismo y biocentrismo. Debates sobre la Naturaleza como sujeto de derechos (parte I). Diario Ambiental Nro 214.

Naciones Unidas. Consejo Económico y Social. E/CN.3/2016/2/Rev.1*. (2016). Informe del Grupo Interinstitucional y de Expertos sobre los Indicadores de los Objetivos de Desarrollo Sostenible.

Ost, F. (1996). Naturaleza y Derecho. Para un debate ecológico en profundidad. En F. Ost, Naturaleza y Derecho. Para un debate ecológico en profundidad (J. A. Irazabal, \& J. Churraca, Trads., pág. 333). Bilbao: Mensajero.

Proyecto Educativo Institucional (2016-2020). Universidad Nacional de Pilar.

Proyecto Educativo Institucional (2016-2020). Facultad de Ciencias Aplicadas. Universidad Nacional de Pilar.

Proyecto de la Carrera Ingeniería Ambiental. Insituto de Ciencias Ambientales. Universidad Nacional de Pilar.

Salazar Ortiz, V. H., \& Láriz Durón, J. J. (2017). La discusión en torno al antropocentrismo en la ética ambiental. Varia, 107-130.

Sírvent, M. T., \& Rigai, L. (2012). https://www.flacsoandes.edu.ec/. Recuperado el 23 de abril de 2019, de https://www.flacsoandes.edu.ec/.

Toca Torres, C. E. ( 2011). Las versiones del desarrollo sostenible. Sociedade e Cultura, Goiânia, 195-204.

Vieytes, R. (2004). Metodología de la Investigación en organizaciones, mercado y sociedad. Epistemología y Técnicas. Buenos Aires: Editorial de las Ciencias. 